\title{
Carnets
}

Revue électronique d'études françaises de l'APEF

Deuxième série - 21 | 2021

Arts du vin

\section{Représentations croisées du vin et de l'alcool chez Davodeau et Kundera}

Luís Carlos Pimenta Gonçalves

\section{OpenEdition}

1 Journals

Édition électronique

URL : https://journals.openedition.org/carnets/12985

DOI : 10.4000/carnets. 12985

ISSN : 1646-7698

Éditeur

APEF

Référence électronique

Luís Carlos Pimenta Gonçalves, «Représentations croisées du vin et de l'alcool chez Davodeau et Kundera », Carnets [En ligne], Deuxième série - 21 | 2021, mis en ligne le 31 mai 2021, consulté le 01 juin 2021. URL : http://journals.openedition.org/carnets/12985; DOl : https://doi.org/10.4000/ carnets. 12985

Ce document a été généré automatiquement le 1 juin 2021.

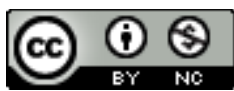

Carnets est mis à disposition selon les termes de la licence Creative Commons - Atribution - Pas d'utilisation commerciale 4.0 International. 


\title{
Représentations croisées du vin et de l'alcool chez Davodeau et Kundera
}

\author{
Luís Carlos Pimenta Gonçalves
}

1 On boit paradoxalement de moins en moins de vin en France, mais on en parle de plus en plus comme le signale Gilbert Garrier dans l'introduction à son Histoire sociale et culturelle $d u$ vin en une jolie formule : « le vin n'est plus sur toutes les tables, mais il est sur toutes les lèvres » (Garrier, 1998: 9). Il est vrai que le vin, et plus généralement les boissons alcoolisées, se donnent à voir ${ }^{1}$ autant qu'à lire dans des guides, magazines et aussi bien dans la littérature et la bande dessinée.

2 Nombreuses sont les œuvres littéraires récentes dont le principal thème est le vin et l'alcool, leur production, mais surtout leur consommation comme c'est le cas de François Vignes, le bien nommé, quand il signe Les compagnons du verre à soif chez Le Cherche Midi en 2002 dont l'incipit révèle la teneur: "Il allait au bistrot comme d'autres vont au travail : avec une ponctualité, une assiduité, propres à susciter les félicitations du plus vétilleux des chefs du personnel » (Vignes, 2002 : 9). Ainsi du belge Jean-Claude Pirotte (2006) avec Expédition nocturne autour de ma cave où se mélangent le goût du vin et l'amour de la littérature et de l'art en une flânerie et une errance dont le titre du livre représente une allusion transparente à l'ouvrage de Xavier de Maistre. Michel Quint avec Les Joyeuses, en 2009, compose un roman d'initiation à la vie, au théâtre, au vin et à son rôle libérateur de parole². Plus récemment, en 2019, Gérard Oberlé signe un recueil de sept nouvelles au titre suggestif Heptaméron avec Chardonnay, publié chez Grasset où, au détour d'une phrase de la nouvelle «Monsieur Justin Galmiche ", il indique que, grâce à J.-C. Pirotte, il avait été initié au savagnin et au chardonnay (Oberlé, 2019:19) et « dégoise » des vers de l'auteur belge dans la nouvelle «Volodia » (Oberlé, 2019: 58).

3 Le vin et le travail de la vigne ne surgissent comme thèmes dans l'univers de la bande dessinée que dans les dernières décennies et c'est dans un pays, le Japon, où il n'y a pas de tradition ancestrale viticole que des mangas s'intéressent à ce sujet. Tout d'abord, 
Sommelier, série en six volumes, créée en 1996 par Araki Joh (scénariste) et Shinobu Kaitani (dessinateur), qui met en scène Joe Satake, Japonais résidant à Paris et $\mathrm{y}$ exerçant la sommellerie. Bien plus étonnant encore est le succès planétaire du manga Les Gouttes de Dieu (Kami no Shizuku) écrit par Tadashi Agi et dessiné par Shū Okimoto entièrement consacré au vin dont les 44 volumes ont été publiés entre 2004 et 2014 . Il s'agit d'un prétexte permettant de découvrir treize grands crus, la plupart français (bordeaux, bourgognes, champagnes...) En France, le scénariste Benoist Simmat ${ }^{3}$ et le dessinateur Philipe Bercovici signent en 2011 un très caustique Robert Parker - Les Sept Péchés Capitaux ${ }^{4}$ qui s'intéresse au créateur du Guide Parker, critique influent de la planète vin dont parle également de façon très critique le film documentaire de 2004 Mondovino ${ }^{5}$. Robert Parker qui est considéré, à tort ou à raison, comme le " plus grand dégustateur du monde » a exercé une influence déterminante sur la vinification des grands vins et leur commercialisation. Figure qui sera évoquée de façon bien plus nuancée dans le roman graphique Les Ignorants par le vigneron Richard Leroy. Le scénariste et dessinateur, Étienne Davodeau, lui fait ainsi dire : "Parker n'est pas un imbécile, et il sait bien goûter... en tout cas, certaines régions. Et ce que j'apprécie, chez lui, c'est sa culture mondiale du vin, et sa méfiance de la 'typicite' ${ }^{6}$ " (Davodeau, 2011 : 106). Le chapitre huit de l'album, « New York Montbenault New York », est entièrement consacré à la venue d'un collaborateur de Richard Parker qui goûte différents vins « qui s'activent dans les barriques, puis ceux des années précédentes » (Davodeau, 2011 : 109) murmurant sur son dictaphone ses observations dans un anglais inaudible pour le vigneron qui contemple la scène et tente vainement d'établir un dialogue avec le goûteur ${ }^{7}$.

4 En septembre 2019, Milan Kundera qui s'est cependant éloigné de la vie publique depuis longtemps a reçu, à 90 ans, le prix littéraire Château La Tour Carnet couronnant un écrivain, un philosophe ou un intellectuel pour l'ensemble de son œuvre ${ }^{8}$. Bien qu'associé à un grand cru cultivé sur les terres du manoir qui a abrité autrefois la famille de Michel de Montaigne, la récompense n'est pas forcément attribuée à un auteur qui aurait abondamment parlé de vin et d'alcool dans ses œuvres. Si cette possibilité n'existait pas, le prix accordé à Kundera serait immérité. Pourtant, même si vin et alcool n'occupent qu'une place accessoire dans sa fiction dès l'incipit de la première nouvelle de Risibles amours ${ }^{9}$, l'auteur mentionne une eau-de-vie tchèque à base de prunes fabriquée en Moravie, région natale de l'écrivain: "Verse-moi encore un verre de slivodice $»^{10}$ (Kundera, 2006a : 11). Cette prune sert à célébrer la publication d'une étude dans une revue d'art par un des personnages. Cet acte dès l'incipit des opus de l'écrivain franco-tchèque marque de façon inaugurale la célébration par le vin et par les spiritueux qui parcourt l'ensemble de l'œuvre de Kundera. Tout d'abord dans la troisième nouvelle du recueil Risibles amours, «Le Jeu de l'auto-stop » (1965) où l'alcool participe d'une sorte de joute amoureuse dans le restaurant d'un hôtel situé à Nové Zamky, ville faisant alors partie de l'ancienne Tchécoslovaquie. La nouvelle est entièrement inscrite sous le signe du ludisme et du jeu de rôle où l'alcool sert d'agent libérateur. Un jeune homme propose à la jeune fille qui l'accompagne un apéritif alors qu'il sait qu'elle n'est pas habituée à ce rituel social comme le précise le texte : «Elle n'était guère portée sur l'alcool ; elle buvait un peu de vin et aimait bien le porto " (Kundera, 2006a : 103). Toutefois, par défi elle demande une vodka. Les toasts se suivent jusqu'au dernier qui tient de la parade sexuelle : «cette fois ils burent aux seins de la jeune femme » (Kundera, 2006a : 104). Dans le troisième roman de Kundera La Valse aux adieux, une femme trinque avec son amant à l'enfant qu'elle porte: "Oui, à notre 
enfant», et elle but d'un trait son cognac» (Kundera, 2010: 74). Les personnages kundériens trinquaient jusqu'alors en buvant de l'alcool fort, mais dans la deuxième partie du roman, un vieil américano-tchèque épicurien, Bertlef, s'introduit dans un cercle de convives du restaurant d'une ville d'eaux tchécoslovaque, dédaigne la mauvaise bouteille de vin posée sur la table et en commande une autre bien meilleure ${ }^{11}$. Dans ce dernier roman rédigé en Tchécoslovaquie, entre 1970 et 1971, au ton à la fois amer et empreint en même temps de la légèreté du vaudeville, Bertlef apparaît comme le premier personnage kundérien connaisseur en vins. Le lecteur assiste alors à une véritable dégustation avec son rituel immuable à laquelle ne manquent même pas les appréciations lyriques du dégustateur.

Celui-ci porta son verre aux lèvres, goûta et se tourna vers le patron : «Il est excellent. C'est du 23 ?

- Du 22, rectifia le patron.

- Servez ! » dit Bertlef, et le patron fit le tour de la table avec la bouteille et remplit tous les verres vides.

Bertlef prit son verre entre ses doigts. « Mes amis, goûtez ce vin. Il a la douce saveur du passé.

Savourez-le, comme si vous aspiriez, en suçant un long os à moelle, un été depuis longtemps oublié. Je voudrais en trinquant marier le passé et le présent et le soleil de 1922 au soleil de cet instant » (Kundera, 2010 : 225-226).

Il faudra attendre une quarantaine d'années pour retrouver dans l'œuvre de Kundera, avec son dernier roman La Fête de l'insignifiance (2014), un autre long passage sur la dégustation. Mais alors que celui de La Valse aux adieux traduisait une appréciation euphorique du partage et de la découverte d'un millésime, l'extrait de La Fête de l'insignifiance est dysphorique : «Ramon, qui les observait, eut l'impression d'assister à des funérailles où trois fossoyeurs inhumaient le goût sublime du vin en jetant sur son cercueil la terre et la poussière de leur parlote » (Kundera, 2013:85) ${ }^{12}$. La "parlotte $»^{13}$ dont il est question dans cet extrait correspond à ce que Gilbert Garrier identifie comme une des trois façons de déguster et de commenter un vin. Il la considère comme « un bavardage à prétention poétique ou à sous-entendus grivois pour impressionner ou distraire des auditoires ignares " (Garrier, $1998: 450)$. Il regrette d'ailleurs qu'on la retrouve même dans certaines confréries. À l'opposé, il y a la dégustation du professionnel, « objective et froide » (Garrier, 1998: 451), déroutante pour le profane avec ses notations. Entre les deux, il y a celle de l'amateur « cultivé et curieux » (ibidem) "une dégustation de décomposition en éléments visuels, olfactifs et gustatifs, d'identification et, pour finir, d'appréciation globale du vin" (ibidem). Aux trois sens d'appréciation du vin (vue, odorat et goût) s'ajoute un autre dont on parle moins : l'ouïe saisissant le bruit du bouchon que l'on enlève et le pétillement d'un vin mousseux. Dans Les Cinq sens, Michel Serres ${ }^{14}$ parle longuement d'un vieil Yquem de 1947 découvert avec deux amis dans le restaurant d'un " docte marchand» du nord-est parisien qui éveille les sens qui participent à la dégustation. Cette séquence restera durablement gravée dans leur être et leur mémoire: « Nous avons pris tant de temps pour boire ce verre que nous en parlons encore» (Serres, $2014: 200)^{15}$. Roland Barthes dans «Le vin et le lait», essai de Mythologies, considérait le vin comme étant une "substance de conversion » (Barthes, 1970: 70) qui, selon lui, peut faire «d'un faible un fort, d'un silencieux, un bavard " (Ibidem) et de conclure : "d'où sa vieille hérédité alchimique, son pouvoir philosophique de transmuer ou de créer ex nihilo" (Ibidem). C'est de ce pouvoir de transmutation ou mieux de transformation dont il est le plus souvent question dans maintes pages des ouvrages de fiction de Kundera. On cherchera en vain 
dans son œuvre des passages sur le travail de la vigne, sur ses viticulteurs et acteurs, mais on y trouvera, en revanche, dès ses nouvelles de Risibles amours, rédigées entre 1959 et 1968, à son dernier roman de 2013, La Fête de l'insignifiance, des épisodes où les boissons alcooliques (eau-de-vie, cognac, whisky, bière et vin) ont un effet désinhibiteur. Ainsi, l'infirmière Elisabeth, de Risibles amours, pourtant en service, danse ivre en se déshabillant dans la salle de garde de l'hôpital en présence de collègues médecins, hommes et femmes, afin de séduire, croit-elle, le docteur Havel. Au mieux, l'ivresse sera une conséquence non voulue, au pis il s'agira simplement de se saouler comme en témoigne de nombreux passages de La Plaisanterie, d'abord quand la journaliste Helena veut associer ivresse et plaisir après avoir fait l'amour ou quand l'adolescente Lucie participe à une initiation orgiaque avec six jeunes d'une bande qui avaient apporté un mauvais vin : «Elle s'était associée à la soûlerie avec une soumission aveugle » (Kundera, 2007 : 340) ou encore quand, lors d'une fête morave, il est précisé par le narrateur : «bien qu'aucun stand ne proposât de l'alcool, presque tout le monde me parut soûl » (Kundera, 2007 : 373-374). Dans d'autres œuvres du romancier, l'ivresse est associée à l'écriture et à la poésie citant dans La Vie est ailleurs l'appel de Baudelaire face à l'inexorabilité du Temps dans le poème du Spleen de Paris «Enivrez-vous » : «Il faut être toujours ivre... de vin, de poésie ou de vertu, à votre guise » (Kundera, 1991 : 294) ${ }^{16}$. L'ivresse est également présente chez Pétrarque, Lermontov, Verlaine et Goethe, surnoms donnés par le narrateur du roman Le Livre du rire et de l'oubli à des poètes fréquentant le Club des gens de lettres praguois afin de cacher leurs réelles identités pour les protéger de la répression qui sévissait, en Tchécoslovaquie, en 1977, au moment où Kundera commence à rédiger cette œuvre. Dans L'Immortalité, roman de 1990, Goethe est devenu un personnage de plein droit et non plus un simple pseudonyme, sa relation avec Bettina von Arnim et une conversation imaginaire avec Hemingway occupent toute la deuxième partie du roman. Bettina raconte dans une lettre conservée dans La Correspondance de Goethe avec une enfant, qu'elle publia après la mort du poète, une rencontre de juillet 1824 où Goethe s'absenta six fois pour boire du vin en cachette dans une autre pièce (Kundera, 2005b: 108), la lettre soulignant perfidement une sorte d'alcoolisme invétéré chez l'auteur de Faust que commente Kundera.

6 Barthes dans l'essai déjà cité oppose à l'usage du vin en France, envisagé comme un philtre, celui du whisky dont on recherche l'ivresse. L'essayiste va même jusqu'à affirmer que "croire au vin est un acte collectif contraignant» (Barthes, 1970: 70). Cette croyance n'est cependant pas partagée de la même façon dans tous les pays. En effet, dans son troisième roman rédigé directement en français, en l'an 2000, L'Ignorance, Kundera oppose la bière bue en République tchèque au vin de Bordeaux en un saisissant épisode qui se déploie sur plusieurs pages. Irena, exilé à Paris pendant vingt années, revient à Prague avec des bouteilles de vin français pour en faire profiter ses amies tchèques demeurées au pays, car l'ancienne exilée est, en quelque sorte, détentrice d'un "diplôme de bonne intégration" (Kundera, 2006b: 71) à la société française, pour reprendre l'ironique expression de l'auteur de Mythologies. Le titre du roman de Kundera se trouve expliqué au chapitre deux quand le narrateur disserte sur les étymons du mot nostalgie dans diverses langues européennes, de ses dérivés et équivalents. Pour lui, « Sous cet éclairage étymologique, la nostalgie apparaît comme la souffrance de l'ignorance. Tu es loin, et je ne sais pas ce que tu deviens. Mon pays est loin, et je ne sais ce qu'y passe » (Kundera, 2006b : 11). Le bordeaux d'Irena devrait servir à réduire cette "ignorance» et à sceller ses retrouvailles avec des amies de 
jeunesse : l'absorption de ce breuvage effacerait les années d'absence et en le buvant, celles qui sont restées au pays natal connaîtraient l'exil d'Irena, ses joies et ses souffrances, son adaptation à une autre réalité. Or, écrit Kundera : « En Bohême, on ne boit pas de bon vin et on n'a pas l'habitude de garder d'anciens millésimes » (Kundera, 2006b : 44). D'où la gêne ressentie par les amies de l'héroöne qui rejettent dans un premier temps le bordeaux et lui préfère des chopes d'un demi-litre de bière ${ }^{17}$. Cependant, l'ancienne exilée ne voulant pas ternir cette rencontre ne peut que se reprocher ce choix qui marque trop tout ce qui les sépare et, poursuivant sa pensée, elle s'interroge de façon toute rhétorique: "la bière à laquelle ses invitées ont manifesté leur fidélité n'est-elle pas le saint breuvage de la sincérité ? le philtre qui dissipe toute hypocrisie, toute comédie des bonnes manières?» (Kundera,2006b : 45) Elle se résout donc à absorber la même boisson que ses amies, mais constate amèrement qu'elle n'a plus «le goût de la bière » (Kundera, 2006b: 47), ce qui symptomatiquement anticipe l'impossibilité du « grand retour » dont parle avec ironie Kundera à diverses occasions dans le roman. Par la suite, la vision de ces femmes buveuses de bière va revenir constamment dans les songes et les angoisses d'Irena qui se voit entourée de ses anciennes connaissances «levant des chopes de bière, riant perfidement, l'empêchant de s'échapper " (Kundera, 2006b : 159). Vers la fin du roman, elle raconte à un autre ancien exilé devenu son amant à Prague cet épisode : «Imagine, millésime 1982 ! Et elles, exprès, pour me donner une leçon de patriotisme, elles ont bu de la bière ! Ensuite, elles ont eu pitié de moi et, déjà saoules de bière, elles ont continué avec le vin! » (Kundera, 2006 : 192).

7 Ce simple épisode de L'Ignorance signale bien les différences culturelles du vin et de la bière telles qu'elles existent dans l'œuvre de Milan Kundera. Aux personnages tchécoslovaques de ses premiers romans, buveurs d'alcools forts ou de bières, l'écrivain va leur opposer ceux des romans suivants, Tchèques exilés en France, en Suisse, au Danemark ou Français à partir de 1978 avec Le Livre du rire et de l'oubli, qui ont un usage plus diversifié et plus connaisseur des boissons alcooliques: calvados, cidre, vieux millésime d'armagnac et bien sûr vin, dont une bouteille de bordeaux achetée à Paris chez Fauchon par Brigitte dans L'Immortalité.

8 Étienne Davodeau dans son roman graphique publié par Futuropolis en 2011, Les Ignorants, dont le sous-titre Récit d'une initiation croisée révèle la perspective initiatique, s'intéresse à une double ignorance : celle de la vigne et du vin, celle de la bande dessinée et de l'édition. L'album met en scène le dessinateur lui-même et un de ses amis, le vigneron Richard Leroy. Le propos de l'album se trouve expliqué dans les deux premières vignettes.

- Si je comprends bien, pour faire un bouquin, tu veux venir bosser bénévolement dans mes vignes? ... C'est ça?

- Je veux aussi que tu m'expliques ce qui se passe dans ta cave et que tu m'inities à la dégustation. En échange, tu découvriras la bande dessinée. Je t'amènerai des livres. On ira voir des auteurs... et des vignerons (Davodeau, $2011: 3$ ).

Nous tairons l'initiation à la BD qui n'est pas notre sujet pour nous consacrer uniquement à celle de la dégustation du vin. Nous tairons également les épisodes nombreux sur la taille de la vigne, la plantation d'échalas, la substitution des manquants, le décavaillonnage, les caractéristiques des sols et des ceps, la fabrication des barriques, et les grands principes de la biodynamie qui tient compte des « rythmes planétaires et lunaires » (Davodeau, 2011: 79) et où un mélange de bouses de vache et d'eau tient lieu de fertilisant. La dégustation dans le roman graphique correspond aussi 
bien à des moments de sociabilité dans des restaurants et bar à vins comme l'Enchotte à Paris, disparu depuis la parution de l'album, au domicile des protagonistes, dans le bureau d'éditeur de Futuropolis ou dans l'atelier d'un dessinateur, mais également à l'occasion d'une foire ou d'un salon professionnel du vin (Salon Renaissance à Angers), ou dans une cave par un goûteur, un vigneron ou simplement un amateur. L'album est fait de périples et de voyages et sera l'occasion pour les deux principaux personnages de visiter d'autres domaines et d'autres caves de viticulteurs, faisant au besoin $700 \mathrm{~km}$ en voiture pour aller jusqu'au Domaine de Jean-François Ganevat, famille dans la vigne depuis 1650, qui fabrique des Côtes du Jura, prenant l'avion pour Bastia pour visiter Antoine-Marie Arena et son vignoble de Patrimonio (Davodeau, $2011: 241-242$ ), ou se rendant à Bergerac pour faire la connaissance d'un ancien infirmier anesthésiste, Régis Lansade, et du médecin Robert Saléon-Terras. Ces derniers sont d'anciens membres d'une équipe de Médecins Sans Frontières dans l'Afghanistan en guerre de $1986^{18}$ reconvertis dans la vigne dans les années 2000 et créant par la suite le Domaine des Chemins d'Orient où ils développent un Pécharmant que Richard Leroy et Étienne Davodeau goûtent dans le chai (Davodeau, $2011: 256-258$ ).

Le roman graphique qui commence à la fin de l'automne 2010 s'étend jusqu'à l'été 2011 permettant ainsi de découvrir les diverses étapes du travail de la vigne et de la vinification. Ce continuum est rythmé par la dégustation de vins par le dessinateur qui, par exemple, après avoir goûté une vingtaine de verres, déclare forfait en disant : «j’ai les papilles grillées » (Davodeau, $2011:$ 37). Le dessinateur essaye, aussi bien dans un livre que dans une bouteille, comme il l'affirme, de «sentir la loyauté et le plaisir qu'a pris le gars qui a fait le vin... ou le bouquin. » (Davodeau, 2011 : 48). Davodeau, conseillé par Leroy, peut comparer par ailleurs plusieurs « grands vins » de 1989, certains vendus aux enchères pour plusieurs centaines d'euros (Davodeau, 2011: 139-142), et un saumur, Domaine Melaric Billes de Roche de 2008, qui vaut dans le commerce moins de vingt euros. Le viticulteur constate mi-dubitatif, mi-amer : "Celui-là je ne sais pas si c'est un « grand vin ». Je sais juste que, comme toi, j'ai beaucoup de plaisir à le boire. Il est droit. Il a une belle chair... mais à côté des autres, il est invisible. » (Davodeau, 2011 : 143).

11 Alors que l'invisibilité est d'une certaine manière une ignorance ou méconnaissance de l'autre chez les personnages kundériens -- l'ivresse est souvent dans ces romans le ressort d'équivoques tout autant que de (ré)jouissances -- l'ignorance décrite dans l'album de Davodeau est plutôt celle de métiers que l'on découvre par un long apprentissage, faisant ainsi de cet ouvrage une sorte de Bildungsroman graphique de l'âge adulte ou plutôt un Kunstlerroman ${ }^{19}$.

\section{BIBLIOGRAPHIE}

BARTHES, Roland (1970). Mythologies. Paris : Seuil, coll. « Points Essais », n. 10.

DAVODEAU, Étienne (2011). Les Ignorants : Récit d'une initiation croisée. Paris : Futuropolis. 
GARRIER, Gilbert (1998). Histoire sociale et culturelle du vin, suivie de Les mots de la vigne et du vin.

Paris : Larousse-Bordas.

KUNDERA, Milan (1991). La Vie est ailleurs. Paris : Galimard, coll. « Folio », n. 834.

KUNDERA, Milan (2005a). Le Livre du rire et de l'oubli. Paris: Gallimard, coll. Folio n.. 1831.

KUNDERA, Milan (2005b). L'Immortalité. Paris : Gallimard, coll. « Folio », n.ำ2447.

KUNDERA, Milan (2006a). Risibles amours. Paris: Gallimard, coll. « Folio », n. $\stackrel{\circ}{1702 .}$

KUNDERA, Milan (2006b). L'Ignorance. Paris: Gallimard, coll. « Folio », n.4155.

KUNDERA, Milan (2007). La Plaisanterie. Paris: Gallimard, coll. « Folio », n. $\stackrel{\circ}{ } 638$.

KUNDERA, Milan (2010). La Valse aux adieux. Paris: Gallimard, coll. « Folio », n. ํ 1043.

KUNDERA, Milan (2011). CEuvre I. Préface et biographie de l'œuvre par François Ricard. Paris :

Gallimard, coll. « Bibliothèque de la Pléiade ».

KUNDERA, Milan (2013). La Fête de l'insignifiance. Paris : Gallimard.

OBERLÉ, Gérard (2019). Heptaméron avec chardonnay. Paris : Éditions Bernard Grasset.

PIROTTE, Jean-Claude (2006). Expédition nocturne autour de ma cave. Paris : Stocks.

SERRES, Michel (2014). Les Cinq sens. Paris : Librairie Arthème Fayard/Pluriel.

SIMMAT, Benoist (scénario), BERCOVICI, Philippe (dessin) (2011). Robert Parker. Les sept péchés

capiteux. 12 BIS.

VIGNES, François (2002). Les Compagnons du verre à soif. Paris : Le cherche midi.

\section{NOTES}

1. Les publicités sont à l'inverse de plus en plus contrôlées en France depuis la loi Évin du 10 janvier 1991 - Loi (91-32). Les débats parlementaires houleux bien avant l'adoption amendée du texte sur le tabagisme et l'alcoolisme sont évoqués dans L'Immortalité quand un des personnages dans un "demi-sommeil » entend à la radio qu'un député "a déposé à l'Assemblée un projet d'interdiction de toute publicité pour la bière » (Kundera, 2005b : 137). Or, de façon caustique le roman signale la logique du débat d'alors: «un certain pourcentage de catastrophes est imputable à l'ivresse des chauffards, mais on ne saurait interdire le vin, gloire immémoriale de la France ; une part de l'ivresse publique est due à la bière, mais la bière non plus ne saurait être interdite, parce qu'il y aurait violation des traités internationaux sur la liberté des marchés ». (Kundera, 2005b : 138).

2. Tel l'Expédition nocturne autour de ma cave de Pirotte, cet ouvrage est publié chez Stock dans une série intitulée "Écrivins » dont, sur son site, l'éditeur, pudiquement, ne veut pas reconnaître comme une collection, la confinant dans une sorte de limbe éditorial « Hors collection littérature française ». Pourtant, Luc Jacob-Duvernet dans Nos chers éditeurs - 13 écrivains témoignent emploie le terme apparemment banni en citant Philippe Claudel : «L'attente fébrile, voilà peut-être ce qui caractérise le mieux le rapport au livre. Je l'ai éprouvée quand j'ai entrepris une modeste expérience éditoriale: j'ai lancé chez Stock une petite collection, baptisée "Écrivins ", pour recueillir des textes sur le vin rédigés dans une totale liberté formelle ». La collection avait été lancée en 2004 par Philippe Claudel et se destinait à publier quatre fois par an des textes dont le vin serve de toile de fond ou soit tout simplement présent. 
3. Benoist Simmat sera par la suite le scénariste d'autres albums consacrés au vin dont plus récemment L'Incroyable histoire du vin, album paru en 2018 illustré par Daniel Casanave.

4. Premier volume de La Trilogie Bordelaise qui comptera également les Caves du CAC40 et le Dico vino. Sous prétexte d'une fausse invitation d'une confrérie de tastevins, Robert Parker est jugé dans une cave et accusé d'avoir dévoyé les vins de Bordeaux. Les pièces à charge sont notamment le documentaire de Nossiter dont l'œnologue Michel Roland dit : « Le fameux film 'Mondovino, en 2004, a mis à bas le système. J'en ai pris pour mon grade. Robert aussi » (Simmat, 2011 : 18).

5. Film réalisé par Jonathan Nossiter. Le réalisateur, qui a été un temps sommelier, est un connaisseur du vin et de la vigne. En 2007, le livre Le goût et le pouvoir prolonge le film avec des entretiens avec différents intervenants du secteur (vignerons, cavistes...). Nossiter s'y montre redoutable à l'égard du système Parker qui avec ses guides nivelle les goûts, définit des modes, lance des tendances.

6. C'est ce refus de la typicité et de la classification qui explique partiellement le fait que depuis 2008 les vins de Richard Leroy ne sont plus produits sous l'appellation Anjou, mais Vins de France.

7. Sur le blogue d'un caviste qui a pignon sur rue à côté de Montparnasse au titre évocateur, « Le blogue de Mi-Fugue Mi-Raisin» [https://mifuguemiraisin.com/richard-leroy-4/], il est précisé que le dégustateur trouva le vin de Richard Leroy trop oxydé à cette occasion.

8. Distinction créée par Bernard Magrez, viticulteur bordelais et le journaliste Franz-Olivier Giesbert

9. Ouvrage qui ouvre le premier volume de l'édition définitive de l'œuvre de Kundera dans la collection de la Bibliothèque de la Pléiade.

10. Cette référence introduit d'emblée le lecteur français dans un univers culturel étranger que n'aurait pas l'indication «eau-de-vie» ou la forme elliptique «prune», solutions de naturalisation, comme il le fait parfois, qu'il aurait pu adopter dans l'édition définitive de son œuvre en français qui vaut comme originale aussi bien dans la collection Folio que dans celle de la Bibliothèque de la Pléiade.

11. "'Je viens ici tous les jours en fin d'après-midi pour boire une bouteille de vin'. Il examina l'étiquette de la bouteille posée sur la table: 'Mais certainement du vin meilleur que celui que vous êtes en train de boire.' " (Kundera, $2010: 223$ )

12. Cet extrait qui signale cette « Fête de l'insignifiance se poursuit ainsi : «Caliban était en train de verser du bordeaux dans les verres de trois convives; par ses gestes et ses grimaces, il leur faisait comprendre que le vin était d'une qualité rare. Connaissant les bonnes manières, les messieurs levèrent leurs verres, les réchauffèrent pendant un long moment dans leurs paumes, gardèrent ensuite une gorgée dans la bouche, se montrèrent l'un à l'autre leurs visages qui exprimèrent d'abord une grande concentration, puis une admiration étonnée, et finirent par proclamer à haute voix leur enchantement. Tout cela dura à peine une minute, jusqu'à ce que cette fête du goût soit brutalement interrompue par leur conversation (...) ».

13. Dans le livre d'entretiens Le goût et le pouvoir: Un cinéaste dans le monde du vin, Jonathan Nossiter parle d'un « blabla postmoderne autour du vin ».

14. Qui a perdu dans ses éditions récentes le sous-titre de Philosophie des corps mêlés qui, en 1985, devait être le premier volume d'un projet plus ambitieux.

15. Cette sensation qui perdure malgré les années permet d'autant mieux l'exercice de remémoration par le philosophe : «La tunique du liquide avait pris un or profond, jaune orangé tirant sur le cuivre, nué de reflets roses : couleur sage et intelligente parfumée aux piquants du désir. On aurait dit un fond de chaudron poli par la patience et le temps dans une cuisine flamande, un peu obscure, entre des traverses de bois noir. Le vin luisait comme la paille dans une étable, comme la boîte du compas illumine le quart, la nuit, dans le vent. Le bouchon, solide, passait déjà, un peu, au fluide, le liège brun virait au blond, tout changeait de phase » (Serres, 2014 : 199-200). 
16. Baudelaire, Le Spleen de Paris, XXXIII. Le Livre de Poche, p. 135.

17. Dans un guide intitulé Le Vin à Prague, daté de 2016, Martin Kuciel et Jan Cerovský affirment que « la République Tchèque est avant tout connue comme le paradis de la bière » et ce bien que, signalent ses auteurs : « on y cultive également du raisin depuis plus d'un millénaire ».

18. L'épopée a été mise en images par Guibert Emmanuel et en texte par Lefèvre dans l'album Le Photographe

19. Approximativement de Lewis Trondheim, publié en album par les Editions Cornélius en 1995, cité par Davodeau dans Les Ignorants (p.54-55) est un exemple de ce sous-genre de roman (graphique) d'apprentissage. Le vigneron en parle ainsi : «le livre du gars qui raconte ses états d'âme et qui se dessine avec un bec d'oiseau » (Davodeau, 2011 : 54).

\section{RÉSUMÉS}

Après une brève présentation du thème $d u$ vin et de l'alcool dans la littérature (hyper)contemporaine et la bande dessinée, cet article s'intéressera plus particulièrement aux représentations de l'alcool et du vin chez l'écrivain Milan Kundera, notamment dans son avantdernier roman L'Ignorance (2000), et chez le dessinateur et scénariste Étienne Davodeau dans Les Ignorants, récit d'une initiation croisée (2011) où le vin et le travail de la vigne apparaissent comme les thèmes principaux de ce roman graphique.

After a brief presentation of the theme of wine and alcohol in (hyper)contemporary literature and comics, this article will focus on the representations of alcohol and wine by the writer Milan Kundera, notably in his penultimate novel L'Ignorance (2000), and in the cartoonist and screenwriter Étienne Davodeau in Les Ignorants, a tale of a cross initiation (2011) where wine and the work of the vine appear as the main themes of this graphic novel.

\section{INDEX}

Mots-clés : Davodeau (Étienne), Kundera (Milan), vin (thème littéraire), littérature, roman graphique

Keywords : Davodeau (Étienne), Kundera (Milan), wine (literary theme), literature, graphic novel

\section{AUTEUR}

\section{LUÍS CARLOS PIMENTA GONÇALVES}

Universidade Aberta

Luis.Goncalves[at]uab.pt 